\title{
Artificial soft robotic elephant trunk made with ionic polymer-metal nanocomposites (IPMCs)
}

\begin{abstract}
A novel design of an artificial soft robot similar to motions of an elephant trunk is presented. The proposed soft undulating robot is a combination of several sections. Each section is an assembly of cylindrical/tubular helical IPMC soft robotic manipulators. The IPMC elements are made of bending IPMC cylindrical/tubular helical elements distributed symmetrically around a cylindrical mantle. This paper further introduces some models and special cases the proposed IPMC soft actuators and sensors in cylindrical/tubular helical form. Experimental results relating the bending deformation to the axial deformations of slit cylinders are also presented.
\end{abstract}

Keywords: ionic polymer-metal composites, electro active polymers, electrophoretically, spectacular, osmotic pressures
Volume 5 Issue 4 - 2019

\author{
Ehsan Tabatabaie S, Mohsen Shahinpoor \\ Department of Mechanical Engineering, University of Maine, \\ USA \\ Correspondence: Mohsen Shahinpoor, Biomedical \\ Engineering/Advanced Robotics (BEAR) Laboratories, \\ Department of Mechanical Engineering, University of Maine, \\ Orono, ME, USA, Email shah@maine.edu
}

Received: June 25, 2019 | Published: July 29, 2019

\section{Introduction}

IPMCs or Ionic polymer-metal composites are electro active polymers (EAPs) and are capable of actuation, energy harvesting, and sensing capabilities among other properties. Thus, they are multi-functional smart materials that can be used in biomimetic soft robotics. They normally require a small electric field of a few $\mathrm{V} / \mathrm{mm}$ and generate spectacular bending and flexing under small electric fields and generate transient voltage and currents output due to dynamic interaction with the environment. Environmental dynamics, like wind or ocean waves, makes them generate an output voltage and transient current of a few $\mathrm{mVs}$ for small samples $(10 \mathrm{~mm} \times 40 \mathrm{~mm} \mathrm{x}$ $0.2 \mathrm{~mm}$ ). IPMCs are capable of operating in or under water or other polar liquids, like blood. Due to the imposed electric field, the conjugated cations

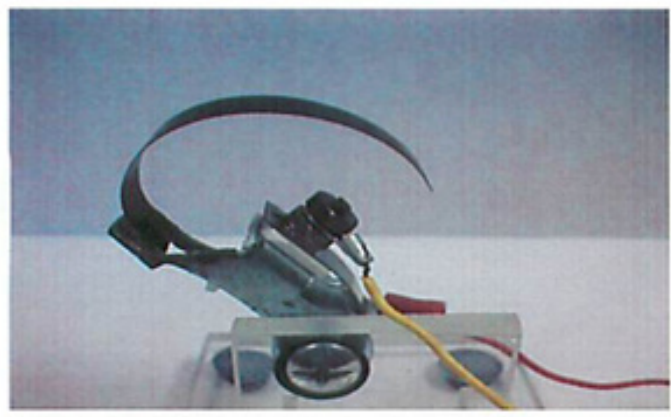

(A) electrophoretically migrate towards the cathode and thus expand the cathode side of the ionic membrane and thus bending towards the anode electrode is observed. Osmotic pressures are also instrumental in the migration of cations towards the cathode electrode. Shahinpoor, Bar-Cohen, Xue, Simpson, and Smith ${ }^{1-2}$ published an early version and review of IPMCs in 1998..$^{1-2}$ See the pioneering works of Osada, Oguro, Kawami, Asaka, Takenaka and Shahinpoor, ${ }^{3-14}$ in the 1992-93periods. The electrodynamics of cations generation and transportation in IPMCs are governed by the PoissonNernst-Planck field equations ${ }^{15-24}$ Biomimetic soft robotic actuation like artificial muscles and sensing of these materials display artificial muscle behavior. For example, an applied voltage of 1 to 4 volts for a typical sample of the size $10 \mathrm{~mm} \times 80 \mathrm{~mm} \times 0.2 \mathrm{~mm}$ typically produces spectacular bending (Figure 1).

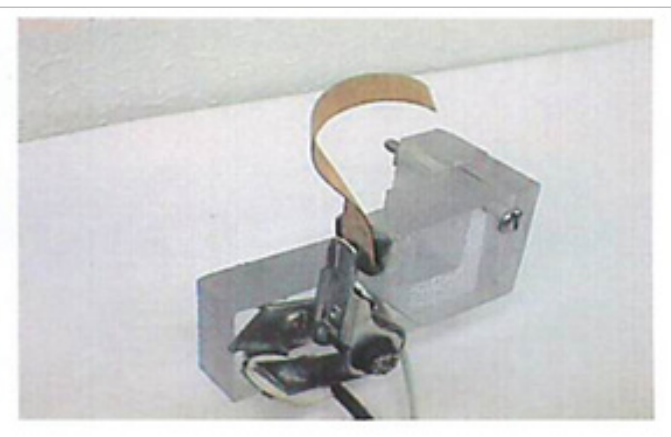

(B)

Figure I IPMC strip spectacular bending under 4 volts (A), IPMC strip bending \& twisting due to the eccentric placement of electrodes (A)

Different kinds of deformation can be generated in IPMCs by various forms of cationic migration under an imposed dynamic electric field across a strip of IPMC. Conversely, physical deformation of IPMCs can generate an output voltage signal, thus serving as energy harvesters and sensors.

\section{Actuation and sensing configurations}

IPMCs, as shown in the following Figure 2, undergo similar cation migrations and rearrangements when subjected to either an electric field or a deformation field. Small samples of IPMC cantilevered strips $(0.5 \mathrm{~cm} \times 3 \mathrm{~cm} \times 0.2 \mathrm{~mm})$ are capable of generating a tip blocking force density of 40 . This capability means they can lift an object 40 times their weight ${ }^{12,23}$ For example, if the weight of an IPMC strip with a density $2 \mathrm{gm} / \mathrm{cm}^{3}$ is $0.1 \mathrm{gmf}$, then it can produce a tip blocking force of about $4 \mathrm{gmf}$.

Several water molecules tend to attach or bond with cations. This number is called the hydration number, 4 for $\mathrm{Na}+$ and 6 for $\mathrm{Li}+$. Poisson-Nernst-Planck phenomena ${ }^{23,24}$ govern such ion dynamics, which we will not dwell upon theoretically in this short paper. 


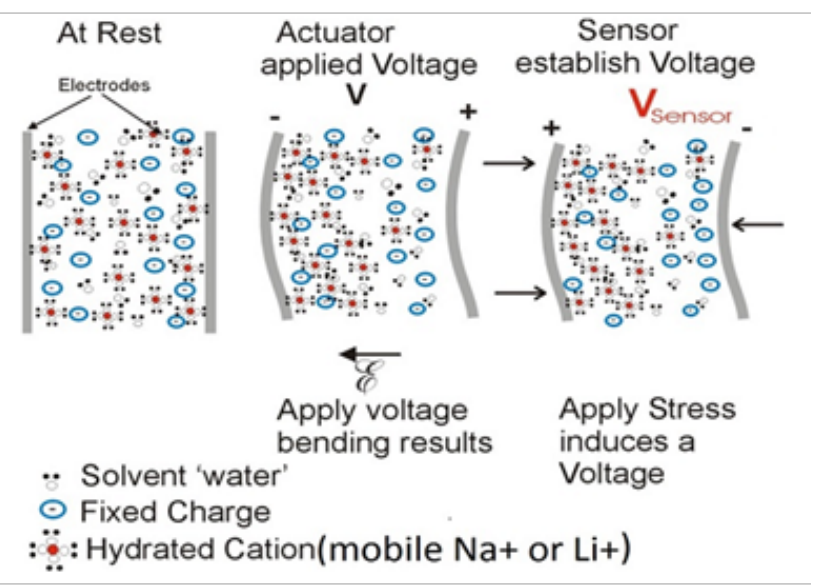

Figure 2 Essential mechanisms of actuation and sensing in IPMCs
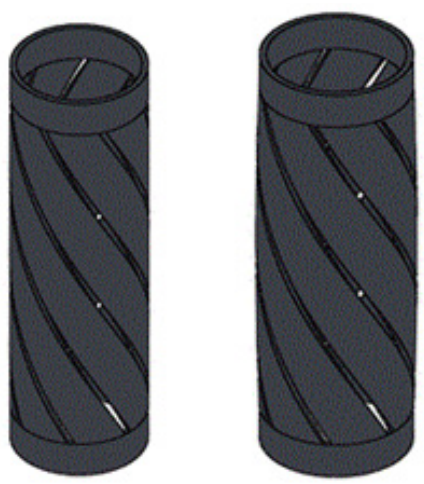

(A)

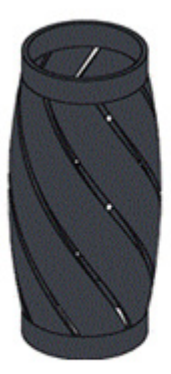

(E)
(B)

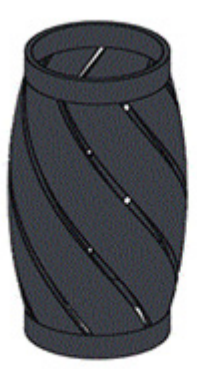

(F)

\section{Artificial soft robotic elephant trunk made with (IPMCs)}

The idea of designing and operating a soft biomimetic highly maneuverable robotic arm like an elephant trunk is highly desirable for many soft biomimetic robotic applications.

If we assemble the IPMC strips bend and rolled around a cylindrical/tubular mantle in the form a slit cylindrical assembly, we begin to observe some highly maneuverable undulation similar to an elephant trunk. Some of the configurations of slit IPMC strips around a cylindrical/tubular mantle are shown below in Figures 3a through $3 \mathrm{~h}$.

The expansion and contraction undulations of the artificial elephant trunk are performed by the elements shown in Figures 3a through $3 \mathrm{~h}$ arranged pair wise in a chain, as shown in Figure 4.

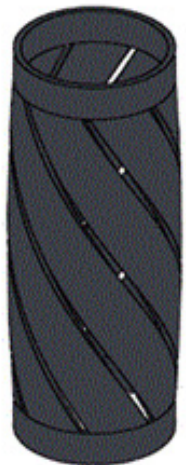

(C)

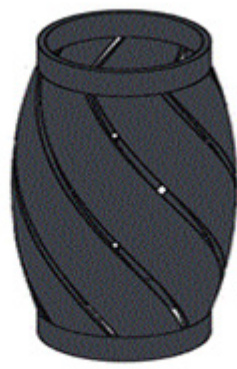

(G)

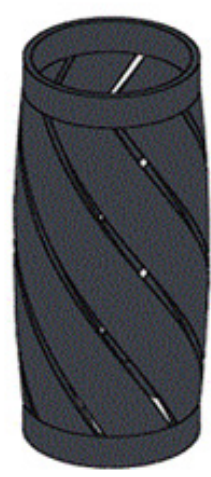

(D)

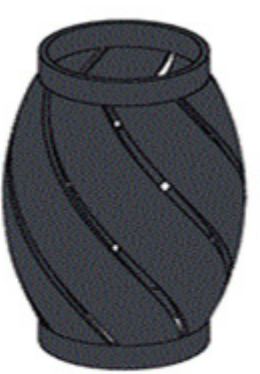

(H)

Figure 3 Basic elements of IPMCs with hinge endings (A), clamped-clamped endings (B)

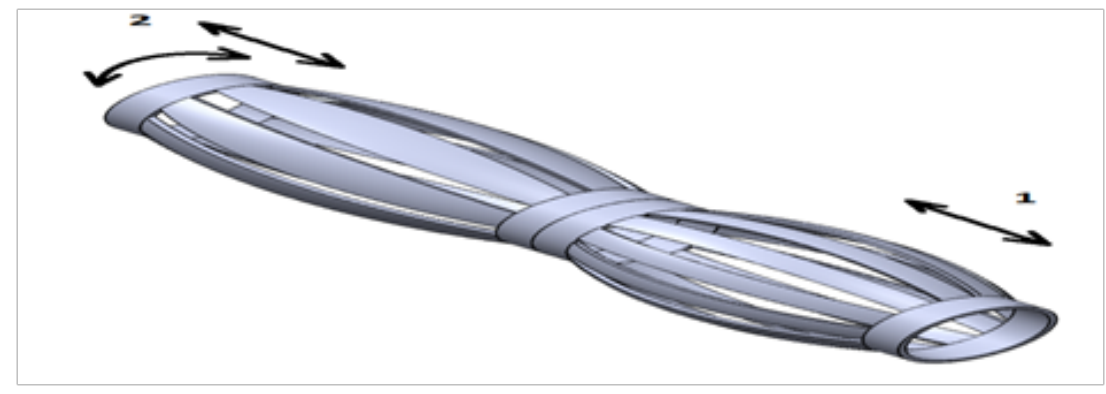

Figure 4 Basic pairs of undulating IPMC strips in a helically slit tubular configuration capable of bending, twisting, expansion, contraction, and undulation. 
In Figure 4 note the combination of movements 1 and 2, the device is capable of 3-D undulating an object like an elephant trunk or a slithering snake-like flexible robotic manipulator.

Note that in the IPMC slit tubular configurations, slit IPMC cylindrical strips have two major forms of boundary condition for this type of the actuator/sensor family in the forms of simple hinges or clamps. Twisting the IPMC slip cylinder then allows for rolling and twisting motion of the slip tubular elements. The twisted and helically wound around the cylindrical mantle will provide more complex manipulation and maneuver possibilities similar to movements of an elephant trunk capable of undulating motion including internal deformation in compression, expansion, twisting, rolling, bending, or twirling.

From another point of views, soft biomimetic artificial elephant trunk robotic actuators and sensors configurations of IPMC as shown in Figures $5 \mathrm{a} \& 5 \mathrm{~b}$, can be employed for the design and fabrication of the biomimetic robotic artificial elephant trunk.

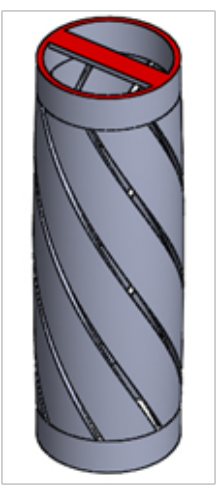

Figure 5A Helical form of slit IPMC actuators and sensors

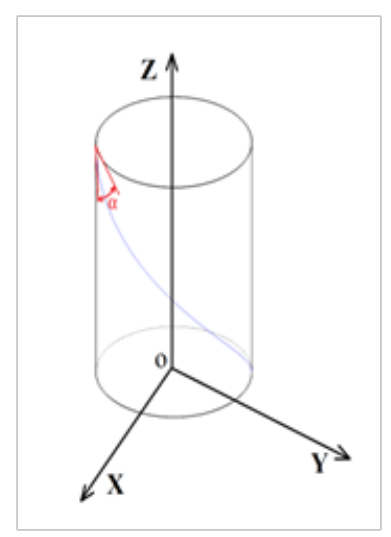

Figure 5B Helical geometry of axial and twisting slit tubular IPMC strips

Consider a rectangular cartesian coordinate systems $\mathrm{x}, \mathrm{y}$ and $\mathrm{z}$ (Figure 5b) such that $x=r \cos \varphi, y=r \sin \varphi$ and $z=c \varphi \operatorname{for} \varphi \epsilon[0,2 \pi]$ . Here $\mathrm{r}$ is the radius of the helix and $\mathrm{c}$ is a constant giving the vertical separation of the helix's loops, and $\varphi$ is an independent parameter. The $\tan \alpha=c / r$ is the value of the slope of the strip sample. The curvature of the helix is given by $\kappa=r /\left(r^{2}+c^{2}\right)$, arc length is given by $s=\varphi\left(r^{2}+c^{2}\right)^{0.5}$, the torsion of a helix is given by $\tau=c /\left(r^{2}+c^{2}\right)$ such that $\kappa / \tau=r / c$.

\section{Soft robotics biomimetic artificial elephant trunk actuators and sensors configurations}

Here the initial form of the device considered for the tubular actuators/sensors is a combination of axial and rotary movements that resemble slithering snakes, as shown in Figure 4 above.

Referring to Figure 6, in case of having more combinations of these actuation configurations, the possibility of having a more complex soft robotic undulating elephant-like trunk can be expected as it is depicted in Figure 6.

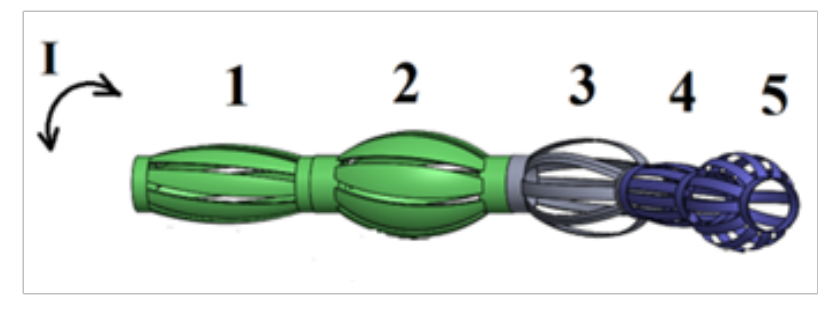

Figure 6A worm/snake/elephant trunk soft robot made of tubular IMPC actuators

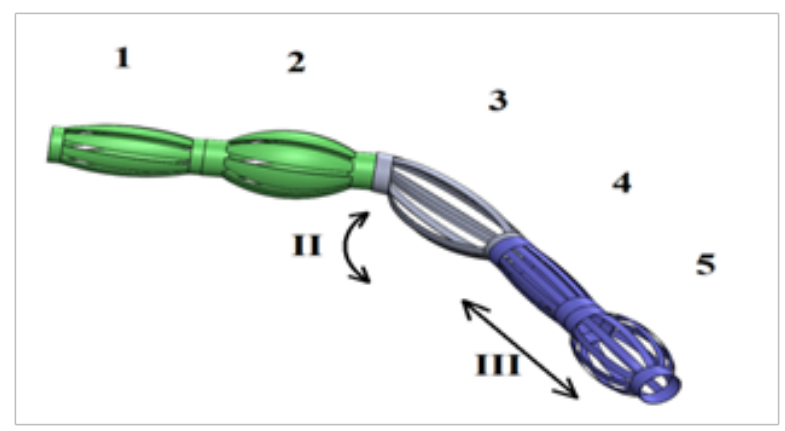

Figure 6B worm/snake/elephant trunk soft robot made of tubular IMPC actuators

Figures $6 \mathrm{a}, 6 \mathrm{~b}$ depict various forms of artificial elephant trunk type undulation with a variety of boundary conditions. Under suitable feedback control, the artificial elephant trunk can be robotically manipulated to move the soft snake-like, worm-like, or elephant trunk-like soft robots. For the rotary movement in the direction denoted by I, actuators 1 and 2 are helically arranged and can produce a combined rotation and slithering movement.

The actuation unit number 3 in Figure $6 \mathrm{~b}$ can produce bending and twisting if we apply a voltage to generate helical and planar movements in the direction denoted by II. Furthermore, other types of undulating movements can be generated by actuator unit number 3 in Figure $6 \mathrm{~b}$ in the direction denoted by III. Thus, the combinations of cylindrically and helically arranged slip IPMC actuators are capable of generating elephant-trunk-like motions and undulations.

\section{Electrical wiring of modular units}

Figure 7 depicts electrical wiring of each modular unit. The anode/ cathode electrodes will be placed either at the proximal, distal, or in the middle of the each IPMC strip to enable moving and controlling each modular unit.

As can be seen in Figure 7, the wire bundle of electrical lead wires is networked to enable IPMC actuation as well as energy harvesting and sensing. 


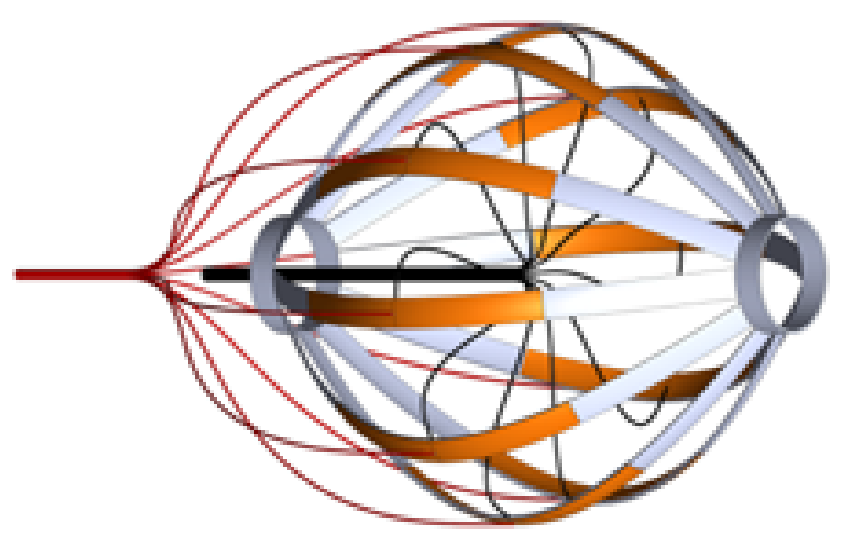

Figure 7 Electrical wiring of each modular unit

\section{Conclusions}

Preliminary design and development of an artificial biomimetic soft robotic elephant trunk were presented. The design used a cylindrical tubular arrangement of helically twisted slit cylinders made with IPMC strips. This family of soft biomimetic artificial elephant trunk robotic actuators and sensors actuator and sensors were designed with bending/twisting slit IPMC elements that were symmetrically arranged and placed around a cylindrical/tubular mantle with either hinged or clamped ends. These types of soft actuators and sensors are capable of generating a combination of actuation, energy harvesting and sensing consisting of linear expansion, contraction, bending, twisting and rotating action similar to a slithering snake or an elephant trunk. The next steps in the development of advanced elephant-trunk-like soft biomimetic robotic actuators and sensors are to further explore various cylindrically and helically arranged IPMC strips. Currently, we are further exploring such possibilities towards fabrication an artificial soft biomimetic robotic elephant-trunk.

\section{Conflicts of interest}

The author declares there are no conflicts of interest.

\section{Acknowledgments}

None.

\section{Funding}

None.

\section{References}

1. Shahinpoor M, Bar-Cohen Y, Simpson JO, et al. "Ionic Polymer-Metal Composites (IPMCs) As Biomimetic Sensors, Actuators and Artificial Muscles-A Review". Smart Materials and Structures. 1998;7(6):R15R30.

2. Shahinpoor M, Bar-Cohen Y, Xue T, et al. "Ionic Polymer-Metal Composites (IPMCs) As Biomimetic Sensors, Actuators and Artificial Muscles". California: Proceedings of SPIE's 5th Annual International Symposium on Smart Structures and Materials. 1998. p. 3324-3327.

3. Osada Y, Okuzaki H, Hori H. A polymer gel with electrically driven motility. SAO/NASA ADS Physics Abstract Service. 1992;355(6357):242244.
4. Oguro K, Kawami Y, Takenaka H. "Bending of an Ion-Conducting Polymer Film Electrode Composite by an Electric Stimulus at Low Voltage". Trans. J Micro-Machine Society. 1992;5:27-30.

5. Segalman D, Witkowski W, Adolf D, et al. "Theory of Electrically Controlled Polymeric Muscles as Active Materials in Adaptive Structures." Int. Journal of Smart Material and Structures. 1992;1:95100.

6. M. Shahinpoor. Conceptual Design, Kinematics and Dynamics of Swimming Robotic Structures Using Ionic Polymeric Muscles. Int. Smart Material and Structures. 1992;1(1):91-94.

7. Doi M, Marsumoto M, Hirose Y. Deformation of Ionic Gels by Electric Fields. Macromolecules. 1992;25:5504-5511.

8. Oguro K, Asaka K, Takenaka H. Polymer film actuator driven by low voltage. Nagoya: In Proceedings of the 4th International Symposium of Micro Machines and Human Science. 1993;38-40.

9. Adolf D, Shahinpoor M, Segalman D, et al. Electrically Controlled Polymeric Gel Actuators. US Patent Office. US Patent No.5,250,167. 1993.

10. Oguro K, Kawami Y, Takenaka H. Actuator Element. US Patent Office. US Patent No. 5,268,082. 1993.

11. Shahinpoor M, Kim KJ. Ionic Polymer-Metal Composites - I. Fundamentals, Smart Materials and Structures. 2001;10(4):819-833.

12. Shahinpoor M, Kim KJ, Mojarrad M. Artificial Muscles: Applications of Advanced Polymeric Nano-Composites. $1^{\text {st }}$ edn. USA: Great Britain: CRC Press, Taylor \& Francis Group. 2007.

13. Kim KJ, Shahinpoor M. Ionic Polymer-Metal Composites - II. Manufacturing Techniques. Smart Materials and Structure. 2003;12(1):65-79.

14. Kim KJ, Shahinpoor M. A Novel Method of Manufacturing ThreeDimensional Ionic Polymer-Metal Composites (IPMC's) Biomimetic Sensors, Actuators and Artificial Muscle. Polymer. 2002;43(3):797-802.

15. De Gennes PG, Okumura K, Shahinpoor M, et al. Mechanoelectric Effects in Ionic Gels. Europhysics Letters. 2007;50(4):513.

16. Asaka K, Oguro KJ. Bending of polyelectrolyte membrane platinum composites by electric stimuli: Part II. Response kinetics. Journal of Electroanalytical Chemistry. 2000;480(1-2):186-198.

17. Shahinpoor M. Ionic Polymer-Conductor Composites as Biomimetic Sensors, Robotic Actuators and Artificial Muscles-A Review. Electrochimica Acta. 2003;48(14-16)2343-2353.

18. Shahinpoor M, Kim KJ. Novel Ionic Polymer-Metal Composites Equipped with Physically-Loaded Particulate Electrode as Biomimetic Sensors, Actuators and Artificial Muscles. Sensors and Actuators A Physical. 2002;96(2-3):125-132.

19. Shahinpoor M, Kim KJ. A Solid-State Soft Actuator Exhibiting Large Electromechanical Effect. Applied Physics Letters. 2002;80(18):34453447.

20. Bahramzadeh Y, Shahinpoor M. Charge Modeling of Ionic PolymerMetal Composites for Dynamic Curvature Sensing. California : Proceedings of SPIE $18^{\text {th }}$ Annual International Symposium on Smart Structures and Materials. 2011. p. 6-10.

21. Bahramzadeh Y, Shahinpoor M. Dynamic Curvature sensing employing Ionic Polymer-Metal Composite Sensors. Smart Materials and Structures. 2011;20(9):7.

22. Shahinpoor M. Biomimetic Robotic Venus flytrap (Dionaea Muscipula Ellis) Made with Ionic Polymer Metal Composites (IPMCs). Bioinspir Biomim. 2011;6(4). 
23. Shahinpoor M. "Ionic Polymer-Metal Composites (IPMCs): Smart Multi-Functional Materials and Artificial Muscles. UK: Volume II, Royal Society of Chemistry Publishers. 2016
24. M.Shahinpoor, "Ionic Polymer-Metal Composites (IPMCs): Smart Multi-Functional Materials and Artificial Muscles". Volume I. UK: Royal Society of Chemistry Publishers. 2016. p. 1. 